\title{
Ginsenoside Compound K Inhibits the Proliferation, Migration and Invasion of Eca109 cell via VEGF- A/Pi3k/Akt Pathway
}

Jianhou Huang

quanzhou first hospital affiliated to fujian medical university

Dinglong Pan

the secend affiliated hospital of fujian medical university

Feng Liu

Dongguan People's Hospital

Yiting Hong

quanzhou first hospital affiliated to fujian medical university

Gang Huang

quanzhou medical college

Xiaowei Huang

quanzhou first hospital affiliated to fujian medical university

Xinwen Wang

Dongguan People's Hospital

Zhiqiang Lin ( $\nabla$ fmulzq@163.com )

quanzhou first hospital affiliated to fujian medical university https://orcid.org/0000-0002-4880-8899

\section{Research article}

Keywords: Ginsenoside CK, Eca109, VEGF-A, knockdown, progression

Posted Date: October 28th, 2021

DOI: https://doi.org/10.21203/rs.3.rs-1004211/v1

License: (c) (i) This work is licensed under a Creative Commons Attribution 4.0 International License.

Read Full License 


\section{Abstract}

Objective: Esophageal cancer, one of the most common cancers of the upper digestive tract and is one of the leading cancer-related mortality worldwide. Accumulating studies found that Ginsenoside compound $\mathrm{K}(\mathrm{CK})$ has significantly anti-tumor effects, especially in the suppression of proliferation, migration, as well as invasion of various human cancers. While the effects of Ginsenoside CK have not well been studied in esophageal cancer. In our present study, we aim to explore the functions of Ginsenoside CK in the progression of esophageal cancer cells (Eca109).

Methods: Cell Counting Kit-8 (CCK-8), wound healing, transwell and flow cytometry assays were applied to analyze the effects of Ginsenoside CK in the progression of Eca109 cell, western bolt was used to investigated the potential downstream signal pathway after Ginsenoside CK treatment.

Results: Our study found that Ginsenoside CK can suppressed the cell viability, proliferation, migration and invasion of Eca109 cell. Furthermore, the flow cytometry showed that Ginsenoside CK induced apoptosis rates of Eca109 cell. The western blot results indicated that Ginsenoside CK functions may possibly related to the blockade of the VEGF-A/Pi3k/Akt signaling pathway. Knockdown of VEGF-A gene could suppress poor the progression of Eca109 cells either.

Conclusion: In conclusion, our study suggests that Ginsenoside CK inhibited viability, proliferation, migration, invasion, and induced apoptosis of esophageal cancer cells by VEGF-A/Pi3k/Akt signaling pathway.

\section{Introduction}

Esophageal carcinoma (EC) is one of the most malignant tumors worldwide, and present a great threat to the health of society [1, 2]. EC is well known by its high rate of metastasis, aggressive invasion and poor prognosis [3]. Surgery and chemotherapy are effective treatments for EC diagnosed at an early stage, a lot of EC patients comes to recurrence or metastasis, and eventually progress to advanced stages of cancers, which present poor prognosis [4]. Therefore, it is of great urgent to explore new and efficient treatment strategies, so as to improve the poor survival status of EC patients.

Ginsenoside CK is the main metabolic component of ginseng in human body [5]. Previous studies demonstrated that Ginsenoside CK has anti-tumor, anti-inflammatory, anti-oxidation, liver protection, improving immune function and other effects [6]. Furthermore, the therapeutics values of Ginsenoside CK in tumors have well been studied in bladder cancer, colon cancer, liver cancer which can significantly inhibited the proliferation ability of these tumor cells [7-9]. Vascular endothelial growth factor-A (VEGF-A), a highly specific pro-vascular endothelial cell growth factor, which can promote extracellular matrix degeneration, vascular permeability, proliferation, migration and angiogenesis of vascular endothelial cells $[10,11]$. Study suggested that ginsenoside CK suppress tumor angiogenesis by suppressing the proliferation and migration of vascular endothelial cells, inhibiting the activity of VEGF-A and it's signaling pathway, and the degradation of vascular extracellular matrix in neuroblastoma 
cells [12]. However, there are few reports about the effects of Ginsenoside CK on esophageal cancer cells and its molecular mechanism. As a targeted drug with high efficiency and low toxicity, ginsenoside CK has further development potential in esophageal cancer.

In our present study, we aim to further explore the effects of Ginsenoside CK on the cell proliferation, migration, invasion and related mechanisms on Eca109 cell.

\section{Materials And Methods}

\section{Cell lines and culture}

Human esophageal cancer cell (Eca109) was purchased from the American Type Culture Collection (Manassas, USA). Eca109 cell maintained in RPMI 1640 medium (Gibco, USA) containing with 10\% feta bovine serum (Gibco, USA) and $1 \%$ penicillin streptomycin (Gbico, USA) in an atmosphere of $5 \% \mathrm{CO}_{2}$ at $37^{\circ} \mathrm{C}$.

\section{Cell viability assay}

The changes of cell viability were evaluated by employing the Cell Counting Kit-8 (CCK-8) assay. Cells (2.5 $\times 10^{3}$ ) per well were cultured in 96-well plate overnight. To investigate the proliferation effect of Ginsenoside CK on Eca109 cell, cells were maintained with ginsenoside CK for $72 \mathrm{~h}$. Similarly, lentivirus transfection cells were maintained in 96-well plate for $24 \mathrm{~h}, 48 \mathrm{~h}, 72 \mathrm{~h}$ or $96 \mathrm{~h}$, then added with 10ul CCK-8 reagent (New Cell \& Molecular Biotech, China) to each well, and then incubated for $2 \mathrm{~h}$ at 37 -C. The absorbance value (OD450) was detected by using the microplate reader (Bio-Tek, USA).

\section{VEGF-A knockdown cell line}

Eca109 cells were transfected with knockdown lentivirus sh-VEGF-A, and the corresponding negative control lentivirus sh-NCwhich were purchased from Hanbio (Shanghai, China). Puromycin was used to screen the stably transfected cells. Western blot analysis was applied to evaluated the efficiency of lentivirus transfection.

\section{Wound healing assay}

Eca109 cell $\left(5 \times 10^{5}\right)$ were seeded in 6-well plates for $24 \mathrm{~h}$ and scraped by a sterile pipette tip. Cells were cultured with DMSO or ginsenoside CK in FBS-free medium. sh-NC and sh-VEGF-A cells were maintained with FBS-free medium. The Zen Imaging software (Carl Zeiss, Germany) was applied to observe images at $0 \mathrm{~h}, 24 \mathrm{~h}$ and $48 \mathrm{~h}$. The Image $\mathrm{J}$ software (USA) was employed to calculate the scratch area.

\section{Cell migration and invasion assays}

The migration and invasion assays were employed by transwell chamber (BD, USA) with the presence of Matrigel (BD, USA) for invasion, and absence of Matrigel for migration. Cells $\left(5 \times 10^{4}\right)$ per well pretreat with 
Ginsenoside CK or lentivirus were planted into the upper chamber with $100 \mathrm{ml}$ serum-free medium, and the lower chamber with $600 \mathrm{ml}$ complete medium. After incubation at $37^{\circ} \mathrm{C}$ for $24 \mathrm{~h}$, the lower chamber cells were fixed with $70 \%$ methanol and then stained by crystal violent (Beyotime, China). The migrated or invaded cells were counted.

\section{Flow Cytometry}

Ec109 cells $\left(1.5 \times 10^{5}\right)$ were maintained in 6-well plates at overnight and treated with ginsenoside CK or DMSO for $48 \mathrm{~h}$. sh-NC and sh-VEGF-A cells $\left(1.5 \times 10^{5}\right)$ were planted in 6 -well plates for $48 \mathrm{~h}$. The rates of apoptosis cells were assessed by applying the Annexin V-FITC Apoptosis Detection Kit (Beyotime). The results were detected by the CytoFLEX flow cytometer (Beckman).

\section{Western blot analysis}

Membrane and Cytosol Protein Extraction Kit (Beyotime, China) and Bicinchoninic Acid Protein Assay Kit (Beyotime) were used for protein extracted and quantified. Proteins were separated on $10 \%$ SDS polyacrylamide gel (Beyotime) and then blotted onto PVDF membranes (Millipore, USA). The PVDF membranes were incubated in $5 \%$ skim milk for $2 \mathrm{~h}$. After that, all membranes were cultured with the primary antibodies of anti-Tubulin (Affinity, USA), anti-VEGF-A (Affinity), anti-Pi3k (Affinity), anti-P-Pi3k (Affınity), anti-Akt (Affinity) and anti-P-Akt (Affinity) overnight at $4{ }^{\circ} \mathrm{C}$. The membranes were then incubated with corresponding secondary antibody (Affinity) for $1 \mathrm{~h}$. The protein bands were finally detected by chemiluminescence detection system (ProteinSimple, USA).

\section{Statistical analysis}

SPSS 24.0 software (SPSS Inc., Chicago, USA) was applied to data analysis, presented as mean \pm SD. The difference between two groups were analyzed by Student's t-test. $P$ value less than 0.05 was defined as statistically significant.

\section{Results}

\section{Ginsenoside CK suppress cell viability, proliferation, migration and invasion of Eca109}

After the incubation with Ginsenoside CK for $72 \mathrm{~h}$ in Eca109 cell, the cell viability was decreased with the increased concentration of Ginsenoside CK, as shown in Figure 1. Moreover, wound healing and transwell assay indicated that both the migration and invasion abilities were reduced notably in Eca109 cell after the treatment of Ginsenoside CK (Fig. 2A, B).

\section{Knockdown of VEGF-A gene suppress cell viability, proliferation, migration and invasion of Eca109}

Eca109 cell were transfected with VEGF-A knockdown lentivirus (sh-VEGF-A) or the corresponding negative control lentivirus (sh-NC) (Fig. 3A). The western blot analysis confirmed that Eca109 cell were stably transfected with lentivirus (Fig. 3B). The CCK-8 assay showed that cellviability is remarkably 
reduced after sh-VEGF-A transfection (Fig. 3C). Wound healing and transwell assay further confirmed that VEGF-A gene knockdown suppress the migration and invasion of Eca109 cell (Figs. 4A, B).

\section{Ginsenoside CK inhibition and knockdown of VEGF-A gene promote apoptosis in Eca109 cell}

To investigate the function of Ginsenoside CK inhibition and VEGF-A gene knockdown in cell apoptosis, Annexin V-FITC and PI staining was applied. The flow cytometry analysis of apoptosis showed that Ginsenoside CK inhibition and VEGF-A gene knockdown promoted the apoptosis rate of Eca109 cell (Fig. $5)$.

\section{Ginsenoside CK influences Eca109 cell progression via VEGF-A/Pi3k/Akt pathway}

It is confirmed that the VEGF-A/ Phosphoinositide 3-kinase (Pi3k)/protein kinase B (Akt) signaling pathway play important roles for the tumor progression on proliferation, migration, and invasion behaviors [13]. The present study detected the expression of VEGF-A/Pi3k/Akt pathway related proteins in Eca109 cell by Ginsenoside CK inhibition and VEGF-A knockdown. The results found that the expressions of VEGF-A, P-Pi3k, and P-Akt proteins were obviously reduced in both the inhibition and knockdown groups, while the total proteins of Pi3k and Akt were not changed (Fig. 6), which suggested that the VEGFA/Pi3k/Akt pathway may present as downstream of Ginsenoside CK inhibition in Eca109 cell.

\section{Discussion}

Esophageal cancer is a highly malignant digestive tract tumor, ranking the 5th and 8th among the causes of cancer-related death in worldwide [14]. Although the diagnostic techniques and treatment methods for esophageal cancer have been continuously improved recently, the overall survival status of esophageal cancer patients is still not ideal due to the characteristics of early invasion and distant metastasis [15]. Ginseng, as a treasure of human pharmaceutical culture, contains a variety of active components, among which ginsenoside is the main bioactive compound in ginseng and has broad implications in human disease [16]. Ginsenoside CK is a natural diol-type ginsenoside with medicinal activity in vivo [17]. Studies found that Ginsenoside CK has inhibiting effects on various tumor cells, containing lung cancer, colon cancer, and liver cancer, as well as bone tumor [18-20]. The anti-tumor effects of Ginsenoside CK are mainly reflected in its ability to reduce the proliferation, migration, and invasion in tumor cells [21]. Chen et al. [22] Study found that Ginsenoside CK can induce cell apoptosis and inhibit the biological activities of human osteosarcoma cells via blocking the Pi3k signaling pathway. Oh et al. [23] revealed that Ginsenoside CK promote cell autophagic and apoptosis which can inhibit human neuroblastoma cells viability both in vitro and vivo. In the present study, we investigated the anti-tumor effect of ginsenoside CK and its related mechanism in Eca109 cell. Our study found that Ginsenoside CK can suppress Eca109 cell viability, proliferation, migration, invasion and induce apoptosis via inhibition of VEGF-A signal pathway. Moreover, knockdown of VEGF-A gene by lentivirus transfection confirmed the suppression of proliferation, migration, invasion ability, as well as promote cell apoptosis of esophageal cancer cells. Furthermore, the western blot results revealed the decrease expression of VEGF-A, P-Pi3k, and P-Akt proteins after Ginsenoside CK intervention and VEGF-A knockdown. 
VEGF-A playing as an important regulator of angiogenesis and presenting as the mediator of endothelial cells proliferation [24]. Pi3k/Akt signaling pathway can be activated by angiogenesis inducers and growth factors, like angiopoietins and VEGF-A [25]. Some studies found that Pi3k/Akt presented as the main downstream signaling pathway mediating the biological effects of VEGF-A, and the VEGF-A/Pi3k/Akt signaling pathway playing significant roles in proliferation, migration and invasion of various cellular processes [26]. In our study, the results of western blot demonstrated the Ginsenoside CK inhibition decrease the expression of VEGF-A, P-Pi3k, and P-Akt proteins. Therefore, we speculated that inhibition of the VEGF-A/Pi3k/Akt signaling pathway may be the main way that ginsenoside CK inhibits cell viability, proliferation, migration, invasion, and induce apoptosis in Eca109 cell. Furthermore, the VEGF-A gene knockdown investigation supported the hypothesis that the therapeutic effect of ginsenoside CK may mainly be via affecting Pi3k/Akt signal activation.

\section{Conclusions}

In conclusion, our present study found that Ginsenoside CK can suppress the cell proliferation, migration, invasion, and induce apoptosis of Eca109 cell via VEGF-A/Pi3k/Akt signaling pathway, which suggests that Ginsenoside CK may serve as an effective treatment in EC.

\section{Abbreviations}

EC: Esophageal carcinoma; CCK-8: Cell Counting Kit-8; Ginsenoside CK: Ginsenoside compound K; VEGFA: Vascular endothelial growth factor-A.

\section{Declarations}

\section{Authors' contributions}

LZ and WX responsible for the study design. HH performed the experiments and draft the manuscript. PD, $L F, H Y, H G$ and $H X$ participated in the data analysis. All authors have read and approved the final manuscript.

\section{Acknowledgements}

No applicable.

\section{Funding}

This work is supported by Science and technology Bureau of Quanzhou City (2018N052S).

\section{Availability of data and materials}

The data the support the findings of this study are available on request from corresponding author. 


\section{Consent for publication}

Not applicable.

\section{Competing interests}

The authors declare none conflict of interest.

\section{References}

1. Li N, Zhao Z, Liu P, et al. Upregulation of deubiquitinase USP7 by transcription factor FOXO6 promotes EC progression via targeting the JMJD3/CLU axis. Mol Ther Oncolytics, 2021. 20: p. 583595. http://doi.org/: 10.1016/j.omto.2020.12.008.

2. Zhao $Y$, Wang $Y$, Shan $L$, et al. A network meta-analysis for neoadjuvant and adjuvant treatments for resectable squamous cell carcinoma of esophagus. Sci Rep, 2021. 11(1): p. 6800. http://doi.org/: 10.1038/s41598-021-86102-8.

3. Wang Y, Zhang W, Liu W, et al. Long noncoding RNA VESTAR regulates lymphangiogenesis and lymph node metastasis of esophageal squamous cell carcinoma by enhancing VEGF-C mRNA stability. Cancer Res, 2021. http://doi.org/: 10.1158/0008-5472.CAN-20-1713.

4. Ajani J A, D'Amico T A, Almhanna K, et al. Gastric Cancer, Version 3.2016, NCCN Clinical Practice Guidelines in Oncology. J Nat/ Compr Canc Netw, 2016. 14(10): p. 1286-1312. http://doi.org/: 10.6004/jnccn.2016.0137.

5. Qi W, Yan X, Xu X, et al. The effects of cytarabine combined with ginsenoside compound $\mathrm{K}$ synergistically induce DNA damage in acute myeloid leukemia cells. Biomed Pharmacother, 2020. 132: p. 110812. http://doi.org/: 10.1016/j.biopha.2020.110812.

6. Shin K C, Oh H J, Kim B J, et al. Complete conversion of major protopanaxadiol ginsenosides to compound $\mathrm{K}$ by the combined use of alpha-L-arabinofuranosidase and beta-galactosidase from Caldicellulosiruptor saccharolyticus and beta-glucosidase from Sulfolobus acidocaldarius. $J$ Biotechnol, 2013. 167(1): p. 33-40. http://doi.org/: 10.1016/j.jbiotec.2013.06.003.

7. Wang $\mathrm{H}$, Jiang $\mathrm{D}$, Liu J, et al. Compound $\mathrm{K}$ induces apoptosis of bladder cancer T24 cells via reactive oxygen species-mediated p38 MAPK pathway. Cancer Biother Radiopharm, 2013. 28(8): p. 607-14. http://doi.org/: 10.1089/cbr.2012.1468.

8. Chen L, Meng Y, Sun Q, et al. Ginsenoside compound $K$ sensitizes human colon cancer cells to TRAIL-induced apoptosis via autophagy-dependent and -independent DR5 upregulation. Cell Death Dis, 2016. 7(8): p. e2334. http://doi.org/: 10.1038/cddis.2016.234.

9. Zhang J, Jiang Y, Li Y, et al. Micelles modified with a chitosan-derived homing peptide for targeted intracellular delivery of ginsenoside compound K to liver cancer cells. Carbohydr Polym, 2020. 230: p. 115576. http://doi.org/: 10.1016/j.carbpol.2019.115576.

10. Pan C F, Zhang X, Wang J W, et al. Weichang'an Formula Inhibits Tumor Growth in Combination with Bevacizumab in a Murine Model of Colon Cancer-Making up for the Deficiency of Bevacizumab by 
inhibiting VEGFR-1. Front Pharmacol, 2020. 11: p. 512598. http://doi.org/:

10.3389/fphar.2020.512598.

11. Wang $\mathrm{N}$, Chen $\mathrm{Y}$, Shi $\mathrm{C}$, et al. CREB3L4 promotes angiogenesis and tumor progression in gastric cancer through regulating VEGFA expression. Cancer Gene Ther, 2021. http://doi.org/: 10.1038/s41417-021-00305-9.

12. Park $\mathrm{D}$ and Yoon $\mathrm{M}$. Compound $\mathrm{K}$, a novel ginsenoside metabolite, inhibits adipocyte differentiation in 3T3-L1 cells: involvement of angiogenesis and MMPs. Biochem Biophys Res Commun, 2012. 422(2): p. 263-7. http://doi.org/: 10.1016/j.bbrc.2012.04.142.

13. Wen $\mathrm{N}$, Guo B, Zheng $\mathrm{H}$, et al. Bromodomain inhibitor jq1 induces cell cycle arrest and apoptosis of glioma stem cells through the VEGF/PI3K/AKT signaling pathway. Int J Oncol, 2019. 55(4): p. 879895. http://doi.org/: 10.3892/ijo.2019.4863.

14. Ding $\mathrm{H}, \mathrm{Xu} \mathrm{J}, \mathrm{You} J$, et al. Effects of enteral nutrition support combined with enhanced recovery after surgery on the nutritional status, immune function, and prognosis of patients with esophageal cancer after Ivor-Lewis operation. J Thorac Dis, 2020. 12(12): p. 7337-7345. http://doi.org/: 10.21037/jtd-20-3410.

15. Jia $X$, Huang $C, H u$ Y, et al. Cirsiliol targets tyrosine kinase 2 to inhibit esophageal squamous cell carcinoma growth in vitro and in vivo. J Exp Clin Cancer Res, 2021. 40(1): p. 105. http://doi.org/: 10.1186/s13046-021-01903-z.

16. Sarhene M, Ni J Y, Duncan E S, et al. Ginsenosides for cardiovascular diseases; update on pre-clinical and clinical evidence, pharmacological effects and the mechanisms of action. Pharmacol Res, 2021. 166: p. 105481. http://doi.org/: 10.1016/j.phrs.2021.105481.

17. Guo Y P, Shao L, Wang L, et al. Bioconversion variation of ginsenoside CK mediated by human gut microbiota from healthy volunteers and colorectal cancer patients. Chin Med, 2021. 16(1): p. 28. http://doi.org/: 10.1186/s13020-021-00436-z.

18. Yang L, Zhang Z, Hou J, et al. Targeted delivery of ginsenoside compound K using TPGS/PEG-PCL mixed micelles for effective treatment of lung cancer. Int J Nanomedicine, 2017. 12: p. 7653-7667. http://doi.org/: 10.2147/IJN.S144305.

19. Pak J N, Jung J H, Park J E, et al. p53 dependent LGR5 inhibition and caspase 3 activation are critically involved in apoptotic effect of compound $\mathrm{K}$ and its combination therapy potential in HCT116 cells. Phytother Res, 2020. 34(10): p. 2745-2755. http://doi.org/: 10.1002/ptr.6717.

20. Muthukumar T, Aravinthan A, Sharmila J, et al. Collagen/chitosan porous bone tissue engineering composite scaffold incorporated with Ginseng compound K. Carbohydr Polym, 2016. 152: p. 566574. http://doi.org/: 10.1016/j.carbpol.2016.07.003.

21. Zhang $Y$, Tong D, Che D, et al. Ascorbyl palmitate/d-alpha-tocopheryl polyethylene glycol 1000 succinate monoester mixed micelles for prolonged circulation and targeted delivery of compound $\mathrm{K}$ for antilung cancer therapy in vitro and in vivo. Int J Nanomedicine, 2017. 12: p. 605-614. http://doi.org/: 10.2147/IJN.S119226. 
22. Chen K, Jiao J, Xue J, et al. Ginsenoside CK induces apoptosis and suppresses proliferation and invasion of human osteosarcoma cells through the PI3K/mTOR/p70S6K1 pathway. Oncol Rep, 2020. 43(3): p. 886-896. http://doi.org/: 10.3892/or.2020.7460.

23. Oh J M,Kim E and Chun S. Ginsenoside Compound K Induces Ros-Mediated Apoptosis and Autophagic Inhibition in Human Neuroblastoma Cells In Vitro and In Vivo. Int J Mo/ Sci, 2019. 20(17). http://doi.org/: 10.3390/ijms20174279.

24. Vion A C, Perovic T, Petit C, et al. Endothelial Cell Orientation and Polarity Are Controlled by Shear Stress and VEGF Through Distinct Signaling Pathways. Front Physiol, 2020. 11: p. 623769. http://doi.org/: 10.3389/fphys.2020.623769.

25. Peng $\mathrm{C}$, Chen $\mathrm{H}$, Li Y, et al. LRIG3 Suppresses Angiogenesis by Regulating the PI3K/AKT/VEGFA Signaling Pathway in Glioma. Front Oncol, 2021. 11: p. 621154. http://doi.org/: $10.3389 /$ fonc. 2021.621154 .

26. Chen C H, Lai J M, Chou T Y, et al. VEGFA upregulates FLJ10540 and modulates migration and invasion of lung cancer via PI3K/AKT pathway. PLoS One, 2009. 4(4): p. e5052. http://doi.org/: 10.1371/journal.pone.0005052.

\section{Figures}
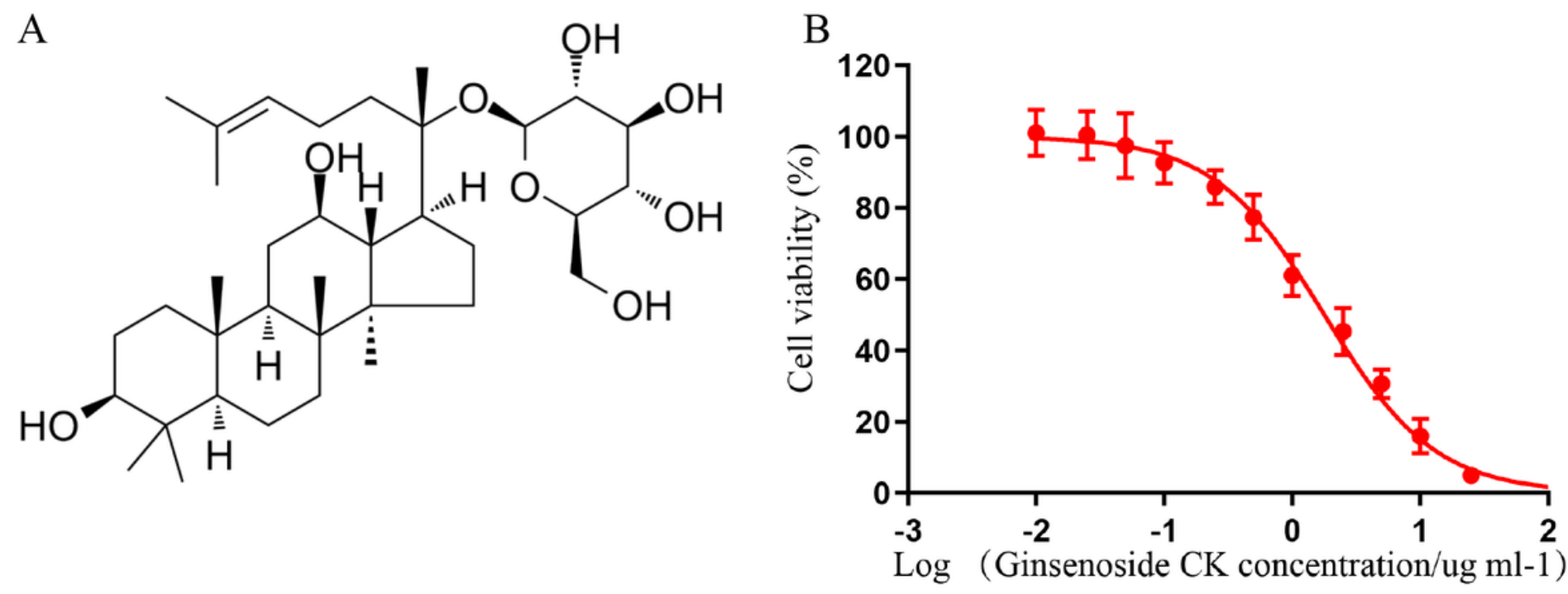

\section{Figure 1}

The drug formula of Ginsenoside CK (A) and cell viability changes with increased concentration of Ginsenoside CK cultured for $72 \mathrm{~h}$ in Eca109 cell (B). 


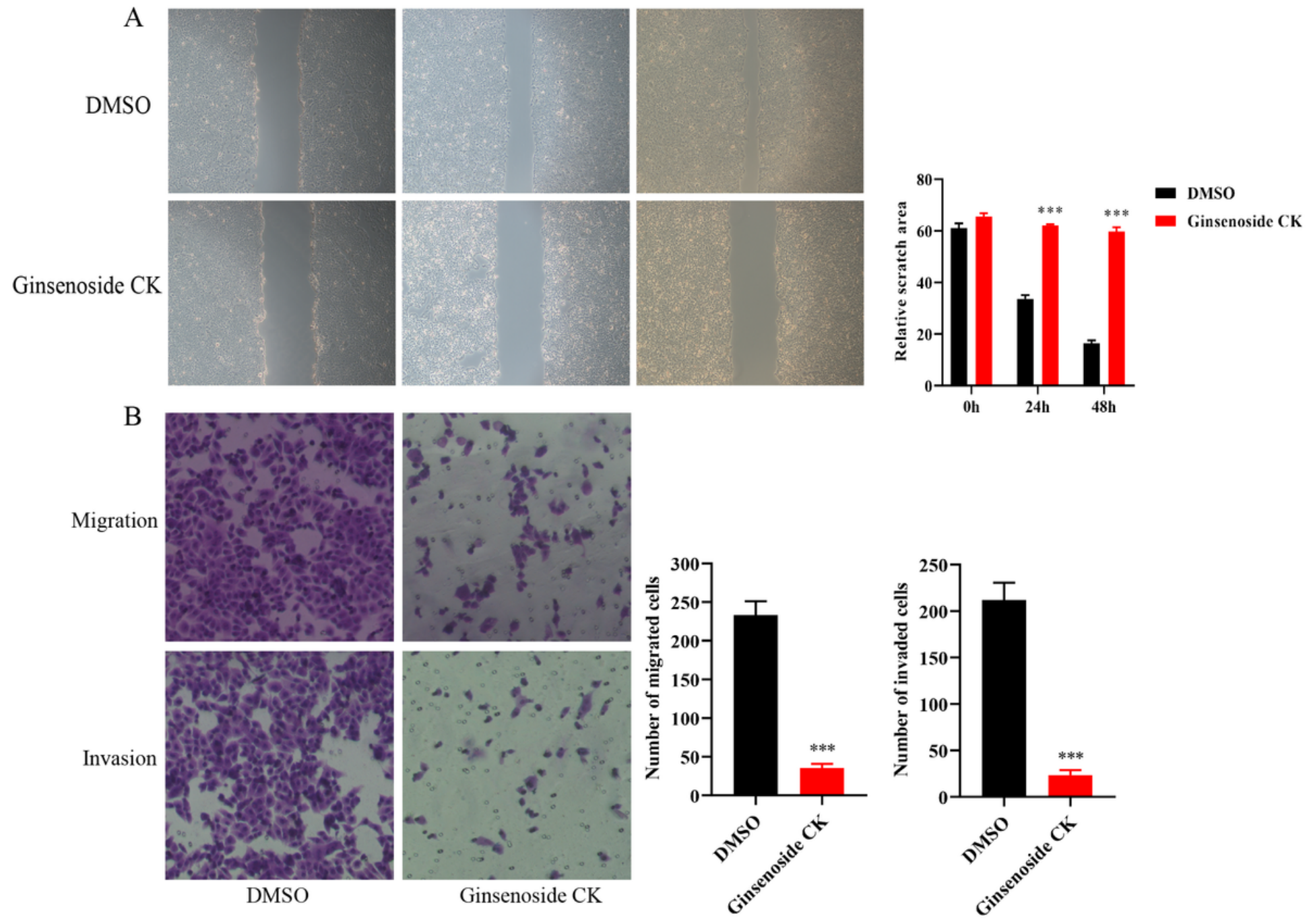

\section{Figure 2}

Ginsenoside CK suppressed the migration and invasion of Eca109 cell. (A) Wound healing assay showed that the migration ability was reduced after Ginsenoside CK treatment. (B) Transwell assay demonstrated that cell migration and invasion ability decreased in Ginsenoside CK intervention. ${ }^{* \star * P \llbracket 0.001}$ 


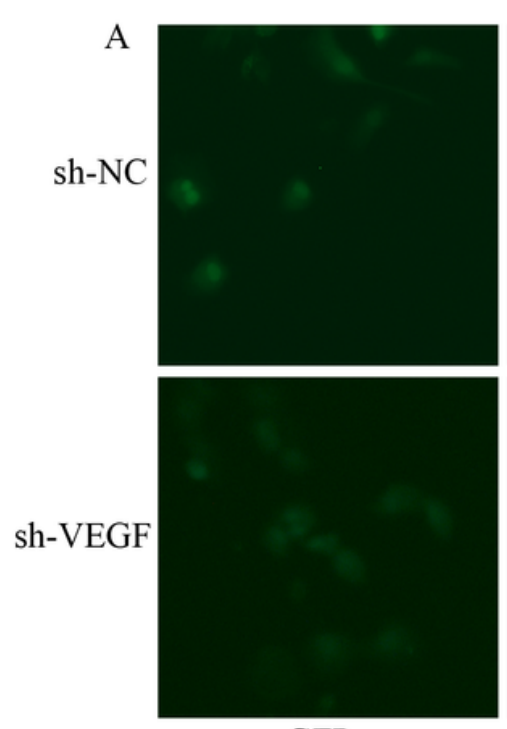

GFP
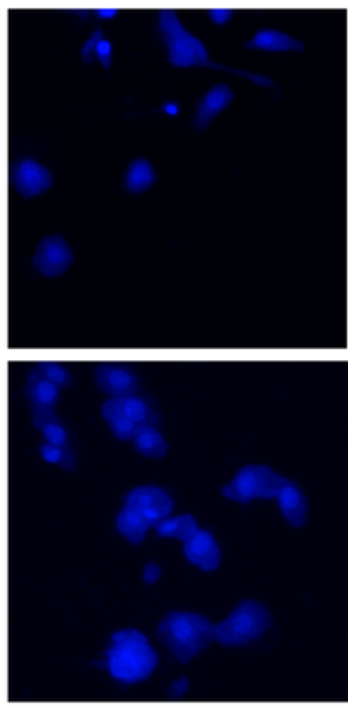

DAPI

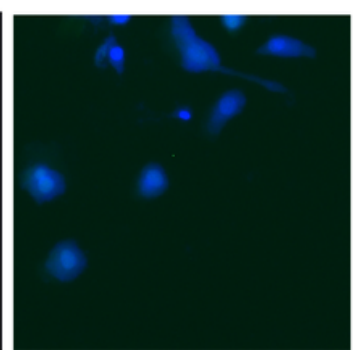

B

VGEF-A

Tubulin

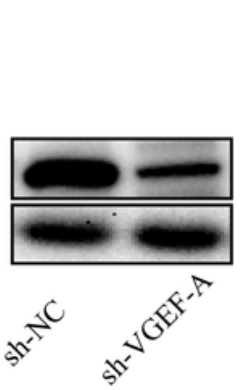

c5
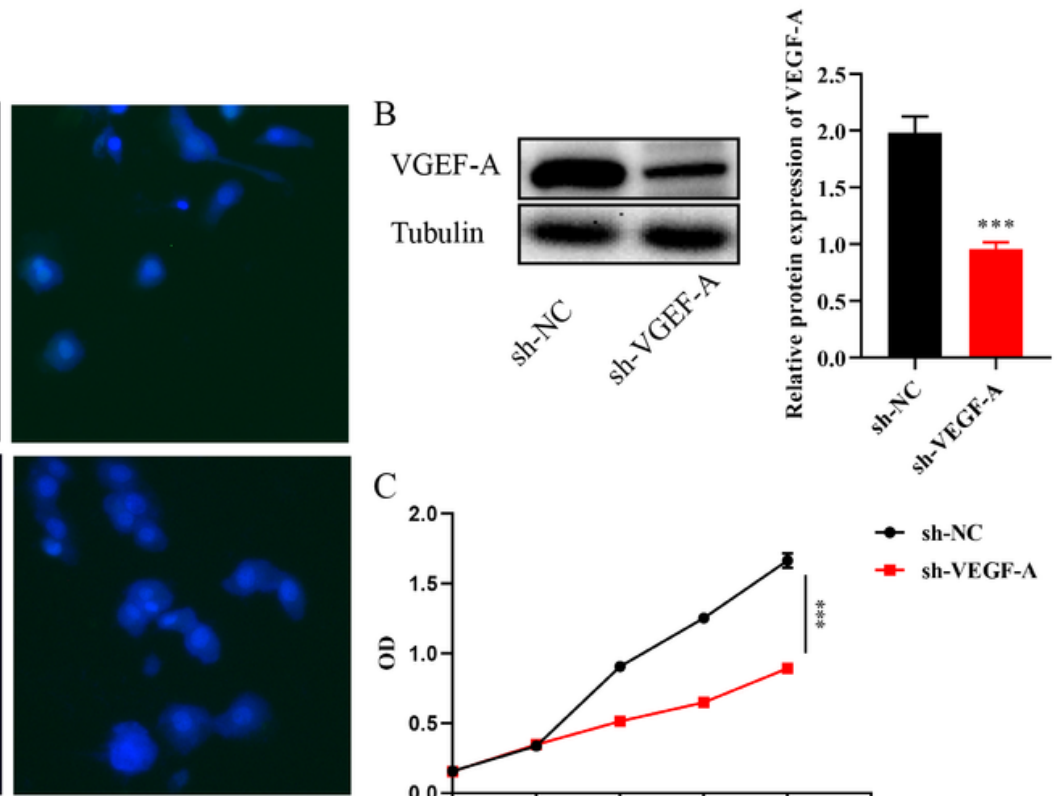

Merge

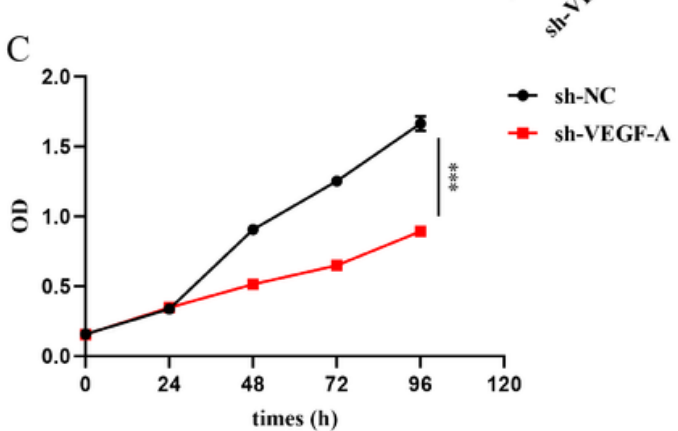

\section{Figure 3}

The knockdown of VEGF-A in Eca109 cell and cell viability changes. (A) Green fluorescent and western bolt analysis showed that Eca109 cell were stably transfected with VEGF-A knockdown gene. (B) Cell viability changes by CCK-8 after incubation for 4 days. *** P $\otimes 0.001$

2

\section{Figure 4}

VEGF-A gene knockdown suppressed the migration and invasion of Eca109 cell. (A) Wound healing assay found that cell migration ability was inhibited after VEGF-A gene knockdown. (B) The migration

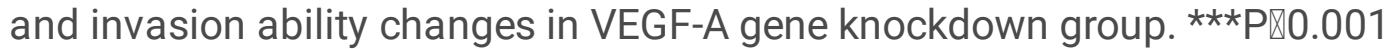



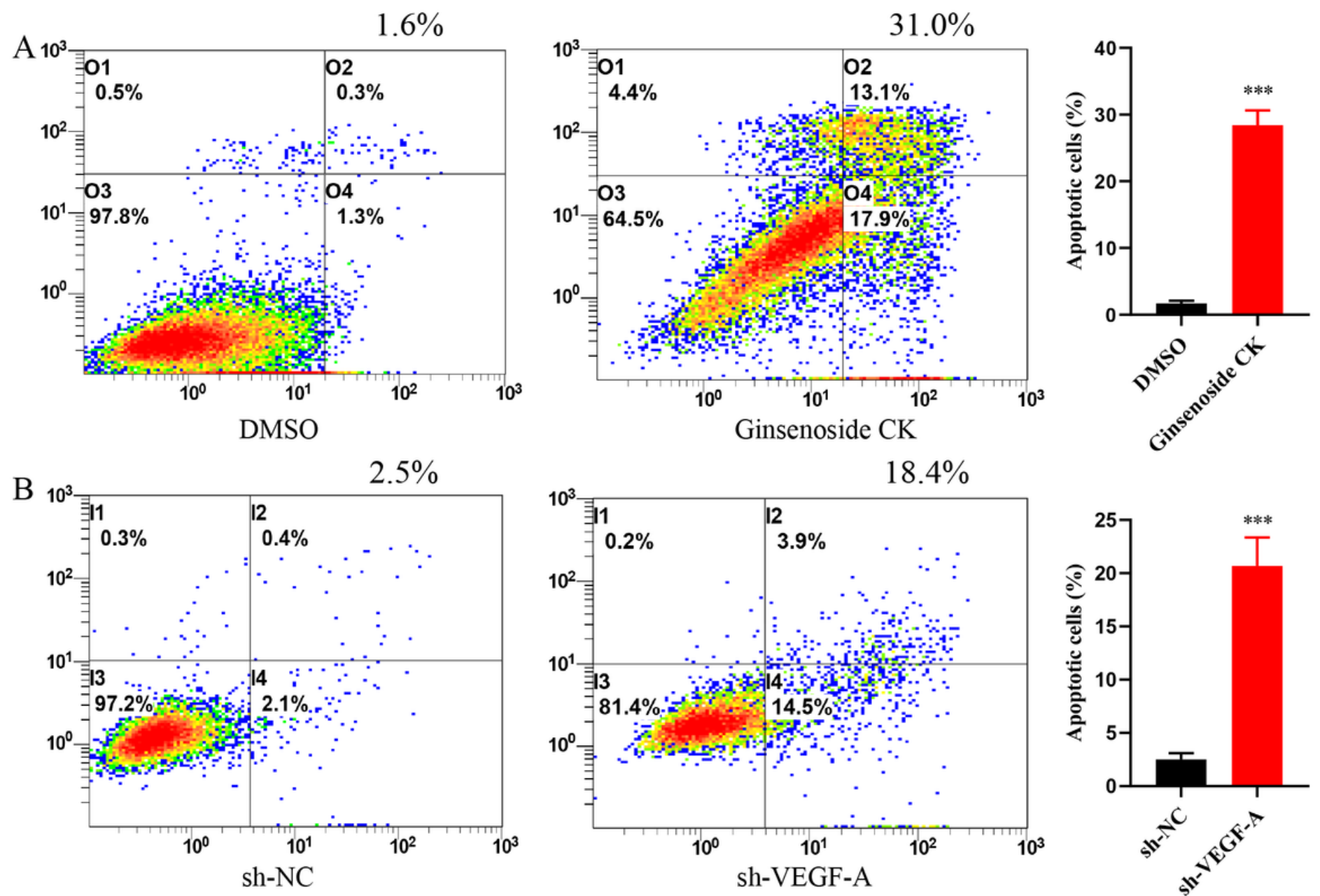

Figure 5

The involvement of Ginsenoside CK and VEGF-A in the pro-apoptotic in Eca109 cell. (A) The cell apoptosis rates of Eca109 cell were detected by flow cytometry with DMSO and Ginsenoside CK

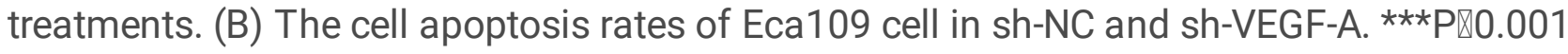




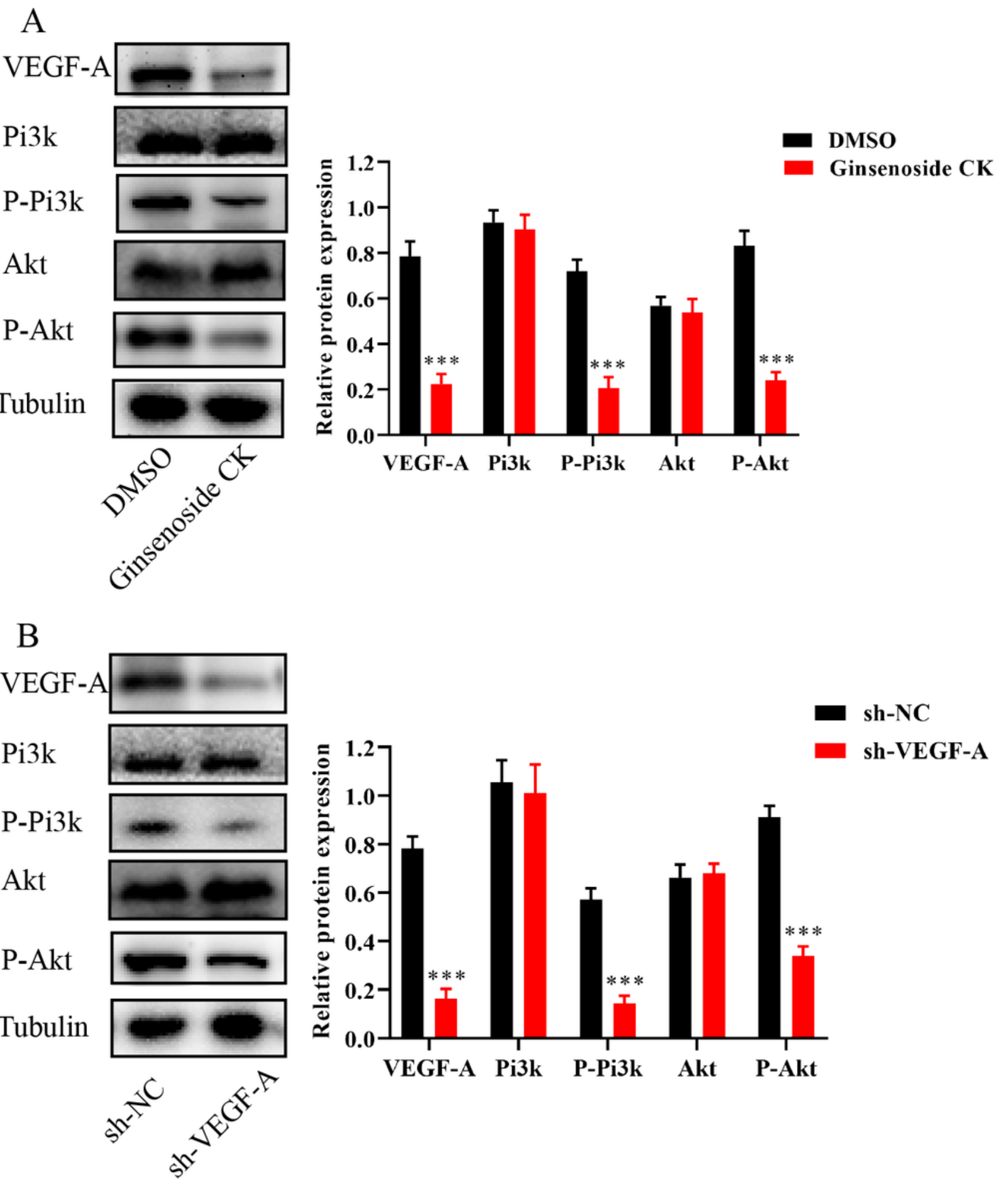

Figure 6

The involvement of VEGF-A/Pi3k/Akt pathway in Ginsenoside CK inhibition and VEGF-A gene knockdown of Eca109 cell. Expressions of VEGF-A, Pi3k, P-Pi3k, Akt, P-Akt in Ginsenoside CK inhibition (A) and VEGFA gene knockdown (B) groups. ***P $₫ 0.001$ 